Stefan Engelberg und Irene Rapp

\title{
Die Gräten einer Harfe. Metaphorische Transformationen und ihre morphosyntaktische Grundlage
}

Sou ons maar 'n metafoor kon vind.

'n Patroon om af de lei.

'n Verhaal om te vertel. ${ }^{1}$

\section{Semantische Konflikte und sprachliche Strukturiertheit}

Es ist geradezu eine Erwartung des Lesers von zeitgenössischen Gedichten, dass er mit Wörtern in sprachlichen Umgebungen konfrontiert wird, in denen er sie zuvor nicht wahrgenommen hat, und dass er ihnen Bedeutungen zuschreiben muss, die nicht seinen lexikalischen Gewohnheiten entsprechen. In vielen solcher Fälle wird der Leser versuchen, sich mit metaphorischen oder metonymischen Interpretationen zu behelfen. Wenn ein Schauspieler als Figur in einem Gedicht „viel Shakespeare ... gespeist und getrunken“ hat, so ist eine naheliegende Interpretation etwa, dass er Shakespeares Dramen (Metonymie) exzessiv gelesen und gespielt hat (Metapher), wobei die kannibalistische Grundbedeutung assoziativ im Hintergrund sichtbar bleibt.

Solche ungewöhnlichen Wortkombinationen und -interpretationen tragen zu dem kreativen, sprachspielerischen Charakter poetischer Sprache bei. Sie erwecken zudem den Eindruck, dass die Sprache in Gedichten abweicht von „normaler“ Sprache. Wir wollen hier nicht unsere Auffassung zu der lange diskutierten Abweichungstheorie poetischer Sprache darlegen (vgl. dazu etwa Levin 1965, Bade \& Beck 2017, Schuster 2017); dennoch sind die folgenden Ausführungen auch ein Beitrag zu dieser Debatte, indem sie zu zeigen versuchen, wie

1 „If we could find a metaphor. Deduce a pattern. A tale to tell.“ (Martjie Bosman: Op die spoor/On the trail. In: Joubert 2014: 34-35). Viele Grüße an Anette und Robert Rosenbach, in deren Tanagra Hill Cottage am Rande der Kleinen Karoo dieser Aufsatz im Juli 2018 entstanden ist. 
bestimmte morphosyntaktische Strukturen die Interpretation kreativer Metaphern unterstützen.

\section{Transformation Richard III}

Ausgangspunkt für unsere Überlegungen ist das Gedicht „Transformation Richard III" von Yoko Tawada. ${ }^{2}$ Im Zentrum des Gedichts steht ein Schauspieler, der die Rolle Richards III. in Shakespeares gleichnamigem Drama spielt.

Transformation Richard III

Ein Seeteufel hängt seine zackige Flosse nach oben Transparent die Schuppen Die Gräten einer Harfe So viel Shakespeare hast du gespeist und getrunken Eine Opfergabe für Götter: dein Fleisch und eine Karaffe

Die Theaterbühne ist dein Kostüm gesteinskörnig Die Materie der Kulisse verletzt deine Haut Ein Hemd zum Ausziehen aus Halsketten geknüpft Der Buckel im Netz der Lederriemen ist ein Ich

Obszön und ungeschützt ist das Königreich der Insel Erfolg stammt aus der Geschwulst im Herrscherhirn Vom Gewand der Rede zieht sich zurück der Zungenzipfel In die Stummheit eines Tiers Es trauert nicht gern

Regieren wollte der der hängt und baumelt Einsam Überlebt der Schauspieler den König jeden Abend Das Innere des Schuhs bleibt dunkel im Publikum Deine Nachttieraugen erkennen jedes Gesicht

Den Hintergrund des Gedichts bildet Shakespeares Drama „Richard III.“, in dessen Eingangsmonolog Richard, Herzog von Gloucester, seine körperliche Missgestalt beklagt: ${ }^{3}$

2 Das Gedicht „Transformation Richard III“ ist erschienen in Tawada, Blamberger und Dopieralski (2018).

3 William Shakespeare: Sämtliche Werke in vier Bänden. Band 3. Berlin: Aufbau-Verlag, 1975. Übersetzung von August Wilhelm Schlegel. Online auf Zeno.org am 11.7.2018: http://www.zeno.org/nid/20005690560. 
Entstellt, verwahrlost, vor der Zeit gesandt In diese Welt des Atmens, halb kaum fertig Gemacht, und zwar so lahm und ungeziemend, Daß Hunde bellen, hink ich wo vorbei; [...]

Da er die Festlichkeiten in den Friedenszeiten, in denen die Handlung angesetzt ist, verabscheut und erotische Abenteuer wegen seines Äußeren meidet, beschließt er, sein Leben auf rücksichtslose und bösartige Weise zu führen, mit dem Ziel, die englische Krone zu erringen:

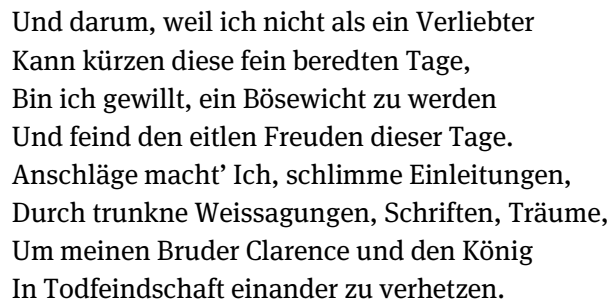

Durch Mord, Gewalt und Intrigen bahnt Richard sich in den folgenden Akten den Weg auf den englischen Thron. Das Drama endet mit dem Tod Richards auf dem Schlachtfeld.

Die in dem Gedicht vorkommenden Metaphern beziehen sich zum größten Teil auf die Transformationen, die dem Gedicht den Namen geben: die Transformation Richards in ein bösartiges Wesen und vor allem die Transformation, der sich der Schauspieler unterzieht, wenn er die Rolle Richards III. spielt.

\section{Die Zweiteiligkeit der Metapher}

Eine metaphorische Interpretation erfordert üblicherweise zweierlei: erstens einen Konflikt und zweitens einen Hinweis, wie dieser Konflikt zu lösen ist. Ein interpretatorischer Konflikt kann entweder dadurch entstehen, (i) dass ein Ausdruck in seinem sprachlichen Kotext nicht zu interpretieren ist, weil seine Bedeutung mit den semantischen Anforderungen der Ausdrücke in seiner unmittelbaren Umgebung unverträglich ist (semantischer Konflikt), oder dadurch, (ii) dass ein Ausdruck im weiteren inhaltlichen Kontext des Gesagten zu textuellen Inkohärenzen führt. ${ }^{4}$

4 Wir verzichten in diesem kurzen Aufsatz auf eine Diskussion der vielfältigen Metapherntheorien. Übersichten dazu finden sich etwa in Rolf (2005) und Knowles und Moon (2006). 
Ein semantischer kotextueller Konflikt entsteht oft dadurch, dass die semantischen Beschränkungen, die ein Wort den von ihm abhängigen Ausdrücken auferlegt, nicht erfüllt werden. Das Verb sich zurückziehen verlangt, dass eine es begleitende direktionale Präpositionalphrase einen Ort spezifiziert, z. B. sich in sein Zimmer zurückziehen. Da Stummheit keinen Ort bezeichnet, ist diese Forderung in zieht sich zurück der Zungenzipfel in die Stummheit eines Tiers nicht erfüllt. Ein semantischer Konflikt entsteht auch in Gräten einer Harfe. Die üblichen Interpretationen für eine attributive Genitivkonstruktion würden unter anderem eine Teil-Ganzes-Relation nahelegen. Es gibt aber keine mereologische Relation zwischen Gräten und Harfen. Ähnlich versagen bei Gewand der Rede die üblichen über die Genitivrelation gesteuerten possessiven oder mereologischen Bedeutungszuordnungen.

Kein unmittelbarer semantischer Konflikt entsteht bei Ausdrücken wie Geschwulst im Herrscherhirn. In anderen Kontexten könnte damit auf einen Tumor Bezug genommen werden. Im gegebenen Gedichtkontext führt das aber nicht zu einer kohärenten Textinterpretation. Der vorliegende kontextuelle Konflikt zwingt uns ebenso wie der semantisch-kotextuelle Konflikt in den obigen Beispielen nach einer vom Üblichen abweichenden Bedeutung zumindest eines der jeweils zwei beteiligten Wörter zu suchen.

Die Lösung solcher Konflikte liegt oft in einer metaphorischen Interpretation für einen der miteinander verbundenen Ausdrücke. Dieser wird von dem Ursprungsbereich, in dem er verortet ist, in einen Zielbereich geführt, wo er eine Bedeutung annimmt, die auf bestimmten semantischen Merkmalen der Ursprungsbedeutung basiert. Zum Beispiel ist der Ausdruck Gewand dem Ursprungsbereich KLEIDUNGSSTÜCKE zugeordnet. Verschiedene semantische (und zum Teil wohl auch enzyklopädische) Eigenschaften sind mit Gewand verknüpft: Ein Gewand ist aus Stoff; es umhüllt, schützt und wärmt den Körper; im Gegensatz zu Klamotten ziert und schmückt es den Träger und hat möglicherweise repräsentative Funktion. Der Hinweis auf die Lösung des Interpretationskonflikts ist nun üblicherweise ein Hinweis auf den Zielbereich der metaphorischen Transformation. Hier kommt er aus dem unmittelbaren Kotext des Wortes: die Rede. Eine metaphorische Interpretation von Gewand würde nun versuchen, ein Konzept im Feld des Oratorischen zu finden, das durch Eigenschaften der Ursprungsbedeutung von Gewand charakterisiert ist. Das wären etwa stilistische Eigenschaften der Rede, die diese in einer den Inhalt nicht verändernden, schmückenden Weise aufwerten. Kontextuell nicht ganz abwegig wäre auch eine Interpretation, in der die verhüllende Schutzfunktion, die Äußerlichkeit und die Repräsentativität des Gewandes hervorgehoben wird: die Rede fungiert als Gewand, indem sie den Redner repräsentativ sozial einbindet und gleichzeitig 
sein Inneres verborgen hält. Im ersten Fall wäre der Genitiv als Possessivus zu interpretieren - das Gewand ist Bestandteil der Rede -, im zweiten Fall als definitorischer Genitiv - das Gewand ist die Rede. ${ }^{5}$ Die beiden metaphorischen Interpretationsvarianten werden zudem dadurch unterstützt, dass die Felder KLEIDUNG/SCHMUCK und REDE/SPRACHE noch durch eine Reihe anderer Metaphern verbunden sind: etwas in Worte kleiden, Redeschmuck, Stoff einer Erzählung und andere. ${ }^{6}$

Dass die metaphorische Interpretation eines Wortes oft mit einem konfliktauslösenden und/oder einem konfliktlösenden Ausdruck verbunden ist, führt dazu, dass sich Metaphern oft in binären Strukturen finden. Hier einige Beispiele aus dem Gedicht Yoko Tawadas:

- Nominalphrase aus Substantiv + Genitiv-NP: Gewand der Rede

- Nominalphrase aus Substantiv + Präpositionalphrase: Geschwulst im Herrscherhirn

- Nominalphrase aus Adjektiv und Substantiv: zackige Flosse (s. dazu Abschnitt 7)

- Substantivkompositum aus Substantiv und Substantiv: Seeteufel

- Verbalphrase aus Verb und Präpositionalphrase: sich in die Stummheit zurückziehen

Einer der beiden Ausdrücke ist jeweils Gegenstand der Uminterpretation; der andere löst den Interpretationskonflikt aus und / oder gibt den Zielbereich der Interpretation an. In Gräten einer Harfe entsteht der Konflikt durch die Unverträglichkeit von Gräten und Harfe und gleichzeitig werden die beiden Bereiche der metaphorischen Übertragung angezeigt: FISCHANATOMIE und MUSIKINSTRUMENTE. Aber woher wissen wir, welches der beiden Wörter nun uminterpretiert wird, und welches die interpretationssteuernde Funktion hat? Grundsätzlich können binäre Ausdrücke dieser Art in beide Richtungen interpretiert werden. Den konkreten Gedichtkontext ignorierend kann Gräten im Bereich MUSIKINSTRUMENTE als 'Saiten einer Harfe' uminterpretiert werden oder Harfe im Bereich FISCHANATOMIE als 'Fischskelett'. Das würde auch für andere binäre Formen gelten wie Harfengräten oder Gräten an einer Harfe. Dass aber nicht alles, was möglich ist, auch sprachlich populär ist, werden wir im nächsten Abschnitt zeigen.

5 Zur Interpretation von Genitivattributen liegt mit der Arbeit von Lindauer (1995) eine umfassende Darstellung vor.

6 Solche Metaphernverbünde werden im Rahmen der konzeptuellen Metapherntheorie von Lakoff und Johnson (1980) behandelt. 


\section{Metaphern und Kopfstrukturen}

Welche der beiden Konstituenten einer zweigliedrigen metaphorischen Struktur eine metaphorische Lesart erhält und welche in ihrer wörtlichen Bedeutung bestehen bleibt, ist durchaus nicht beliebig. Für N+N-Komposita und Nominalphrasen mit Genitivattributen gilt, dass im Defaultfall der determinierte Bestandteil - also der morphosyntaktische Kopf - metaphorisch umgedeutet wird. ${ }^{7}$ Wie wir in Abschnitt 7 zeigen werden, können ko- und kontextuelle Faktoren allerdings bewirken, dass eine Uminterpretation auch beim Nicht-Kopf ansetzt. Derartige Einflüsse wollen wir in diesem Abschnitt außer Acht lassen, d. h. wir betrachten die Metaphern des Gedichts hier zunächst, ohne den weiteren Zusammenhang zu berücksichtigen.

Sehen wir uns zunächst die Genitivmetaphern an. Aus dem Kontext gelöst, tendieren wir stark dazu, Gräte einer Harfe als 'Saite einer Harfe' zu verstehen, d. h. wir interpretieren den syntaktischen Kopf Gräte um. Ebenso geben wir Gewand der Rede vorzugsweise die Bedeutung von 'etwas, das in Bezug auf die Rede ist wie ein Gewand' (s. Abschnitt 3). Die Interpretation von Kompositumsmetaphern funktioniert analog: wir interpretieren Seeteufel als ein Wesen, das Eigenschaften eines Teufels hat, und in Verbindung mit der See (Zielbereich MEER / MEERESLEBEWESEN) steht.

Auf den ersten Blick ähneln sich Genitivmetaphern und Kompositumsmetaphern also stark: beide zeigen eine Präferenz für eine Uminterpretation des morphosyntaktischen Kopfs. Im Folgenden werden wir jedoch zeigen, dass sich Genitivmetaphern wie Gräten einer Harfe und Gewand der Rede auf der einen Seite und Kompositumsmetaphern wie Seeteufel auf der anderen Seite in zweierlei Hinsicht grundlegend unterscheiden: Zum einen signalisieren Genitivkonstruktionen eine metaphorische Interpretation auf deutlich stärkere Weise als Komposita dies tun, zum anderen eignen sich Kompositumsmetaphern wesentlich besser zur Bildung von Ausdrücken, die als Appellativa lexikalisiert werden können. Beides ist darauf zurückzuführen, dass der Nicht-Kopf im Falle von Genitivmetaphern referentiell und diskursbezogen verstanden wird, im Falle von Kompositumsmetaphern dagegen nicht.

7 In Rapp (in Vorb.) wurde dafür plädiert, das syntaktisch orientierte Kopfprinzip („Die metaphorische Uminterpretation betrifft den Kopf“) zugunsten eines semantisch ausgerichteten Prädikatprinzips („Die metaphorische Uminterpretation betrifft ein Prädikat“) aufzugeben. Für die hier diskutierten Beispiele entsprechen sich die beiden Prinzipien; wir verwenden das Kopfprinzip, da es sich in einem nicht-formalen Rahmen leichter illustrieren lässt. 


\section{Die Interpretation von Genitivstrukturen und Komposita}

Die grundlegende Semantik postnominaler Genitive besteht darin, den Referenten des Kopfnomens als zugehörig zum Referenten des Genitivattributs zu kennzeichnen. Illustriert sei dies durch die folgenden Genitivkonstruktionen aus dem vorliegenden Gedicht: das Innere des Schuhs und die Materie der Kulisse. Zu beachten ist, dass beim Attribut tatsächlich Referenz vorliegt: Genitivattribute ohne adjektivischen Modifikator haben immer einen Artikel und nehmen daher Bezug auf etwas, das in den Diskurs eingeführt wird (indefiniter Artikel) oder aber im Diskurs bereits vorhanden ist (definiter Artikel); lediglich bestimmte Abstrakta wie Liebe oder Freiheit bedürfen keiner Diskurseinführung und treten immer mit definitem Artikel auf. Berücksichtigt werden muss bei diesen Überlegungen auch, dass mit vielen Substantiven sowohl auf einzelne Individuen wie auch auf die Gattung Bezug genommen werden kann. Mit das Innere des Schuhs kann ich mich auf einen konkreten Schuh beziehen, über dessen Fütterung ich mich beim Verkäufer erkundige, oder auf Schuhe im Allgemeinen, wenn etwa in einem Lehrbuch für Schuster das Innere des Schuhs behandelt wird. Wichtig ist, dass auch bei Gattungsbezeichnungen das Genitivattribut referentiell ist und somit anaphorisch aufgegriffen werden kann, z. B.: Das Innere des Schuhs ist von Bedeutung, insbesondere wenn wir ihn unter dem Aspekt des Wärmeschutzes betrachten.

Komposita folgen dagegen einem grundlegend anderen Interpretationsmechanismus. Sowohl der Kopf als auch der Nicht-Kopf bezeichnen Eigenschaften, die in eine recht frei zu interpretierende Beziehung gesetzt werden. So fallen unter das Wort Theaterbühne Dinge, die eine Bühne sind und eine Beziehung zu Theater haben. Ein im Diskurs verankertes Theater ist nicht erforderlich; eine Theaterbühne kann etwa auch in einem Depot gelagert sein. Auch der Zungenzipfel muss nicht auf den Teil einer konkreten Zunge referieren, sondern bezeichnet - in alliterierender Abweichung von der konventionalisierten Zungenspitze - ganz allgemein einen Körperteil. Sehr deutlich wird der Unterschied zwischen Genitivkonstruktionen und Komposita im Falle von Halskette, Lederriemen und Nachttieraugen. Halskette drückt in lexikalisierter Bedeutung eine finale Relation aus (Kette für den Hals), Lederriemen eine Beschaffenheitsrelation (Riemen aus Leder) und Nachttieraugen eine Vergleichsrelation (Augen, die so sind wie diejenigen eines Nachttieres). Alle diese Relationen kann die Genitivkonstruktion nicht ausdrücken. Unakzeptabel sind \#Kette des Halses, \#Riemen des Leders und \#deine Augen des/eines Nachttiers. Dabei ist das Beispiel deine 
Nachttieraugen besonders interessant: Im Gegensatz zu Nachttieraugen kann Augen eines Nachttiers keine Vergleichsrelation ausdrücken; deine Augen eines Nachtiers ist eben deswegen inakzeptabel, weil das Genitivattribut eine possessive Zugehörigkeitsrelation ausdrückt und damit eine weitere possessive Zugehörigkeitsrelation (deine) blockiert.

Halten wir fest, dass Genitivkonstruktionen eine grundlegend andere Bedeutung haben als Komposita. Der wesentliche Punkt ist nun, dass ihre Zugehörigkeitsbedeutung genau dem entspricht, was wir für die metaphorische Übertragung brauchen: Ein Objekt (bezeichnet durch das Kopfnomen) wird einem neuen Bereich zugeordnet - dem Zielbereich der Metapher. Anders gesagt, im nichtmetaphorischen Fall nimmt die Genitivkonstruktion eine Zuordnung zu einer Bezugseinheit im nichtübertragenen Sinne vor, z. B. dem Besitzer in einer Besitzrelation oder dem Ganzen in einer Teil-Ganzes-Relation. Wenn dies aufgrund eines Interpretationskonflikts nicht möglich ist, springt die Bedeutung sofort auf eine metaphorische Ebene und ordnet das Kopfnomen einem neuen Zielbereich zu.

Die Unterschiede zwischen Genitivkonstruktionen und Komposita bezüglich ihrer Interpretationspräferenzen seien nochmals an Textbeispielen illustriert. Im Falle von Gewand der Rede liegt eine Genitivkonstruktion vor. Man sucht zunächst einmal nach einer mit den Grundbedeutungen der Wörter verträglichen Zugehörigkeitsbeziehung, die aber nicht zu erschließen ist. Daraufhin wird sofort der Schritt zur Metapher vollzogen, d. h. Gewand wird dem Bereich der Rede zugeordnet und entsprechend uminterpretiert. Beim Kompositum Redegewand wäre dagegen ohne Weiteres eine finale Relation zwischen den beiden Konstituenten denkbar - etwa 'Gewand, das bei Redeanlässen angelegt wird'. Die metaphorische Umdeutung von Gewand ist hier nicht ausgeschlossen, jedoch keineswegs zwingend. Auch bei Gräten einer Harfe ist eine Zugehörigkeitsrelation im nichtübertragenen Sinne nicht möglich; daher weicht die Interpretation bei der Genitivkonstruktion sofort auf die metaphorische Lesart aus. Das Kompositum Harfengräten könnte man dagegen auch interpretieren als 'Gräten für eine Harfe', also - mit etwas Bereitschaft zu technologischer Innovation - z. B. als 'Gräten, die man zur Reinigung einer Harfe verwendet'.

\section{Die Lexikalisierung von Metaphern}

Im vorigen Abschnitt haben wir gesehen, dass Substantive mit attributivem Genitiv immer eine Zugehörigkeitsrelation zu einem Attributsreferenten herstellen. Die Referentialität des Attributs führt nun dazu, dass solche Genitivkonstruk- 
tionen sich bezüglich einer möglichen Lexikalisierung grundlegend anders verhalten als Komposita: Während Komposita ohne Weiteres lexikalisiert werden können - wie dies auch bei Seeteufel, Theaterbühne u. a. der Fall ist -, können Genitivkonstruktionen nicht gut als Mehrwort-Appellativa lexikalisch gespeichert werden. Als feste, lexikalisierte Ausdrücke müssten sie das Genitivattribut für Definitheit und Numerus spezifizieren: Bühne des Theaters würde den Referenten der Genitiv-NP also immer schon auf ein singularisches, in den Diskurs eingeführtes Theater beschränken - eine Einschränkung, die Theaterbühne nicht betrifft. Auch hier gilt allerdings: Steht in der Genitiv-Phrase ein Abstraktum, das nicht erst in den Diskurs eingeführt werden muss und auch nie pluralisiert wird, so sind lexikalisierte Genitivkonstruktionen durchaus möglich, wie etwa das Auge des Gesetzes oder die Dornen der Liebe.

Fest steht, dass Klassenbildung, d. h. eine Lexikalisierung als Appellativum, bei Komposita üblich ist, bei Genitivkonstruktionen jedoch eher selten auftritt. Auch die Beispiele im Text zeigen dies: so sind Seeteufel, Opfergabe, Theaterbühne, Halskette, Lederriemen, Königreich lexikalisierte, teilweise auch idiomatisierte Appellativa, wohingegen die Stummheit eines Tiers und das Innere des Schuhs keinerlei Lexikalisierung aufweisen. Natürlich gibt es auch im Bereich der Komposita okkasionelle, d.h. nicht-lexikalisierte Bildungen (Zungenzipfel, Nachttierauge). Diese lösen jedoch beim Hörer ein lexikalisches Neuheitsempfinden aus. Auch dies zeigt, dass Komposita anders als syntaktische Phrasen im Normalfall lexikalisiert sind. Bei einem Kompositum erwartet man Lexikalisierung und empfindet Neubildungen aus diesem Grunde als auffällig, bei syntaktischen Phrasen - wie der Genitivkonstruktion - gibt es dagegen keine derartige Erwartungshaltung.

Welchen Effekt hat dies nun für die Wirkung metaphorisierter Genitivkonstruktionen? Betrachten wir nochmals die Gräten einer Harfe vs. Harfengräten. Beides kann sich, wie oben gezeigt, auf die Saiten einer Harfe beziehen - eine Lexikalisierung als Appellativum wäre jedoch nur für das Kompositum möglich. Dies wäre ein im Bereich der Musikinstrumente durchaus übliches Muster, wo Ausdrücke für Körperteile oft metaphorisch verwendet werden: Geigenhals, Maultrommelzunge, Jocharm (bei einer Lyra), Stimmwirbel, Harfenfuß, Harfenschulter, Harfenhals, Harfenkrone. Dass Harfengräte dennoch nicht als Appellativum lexikalisiert ist, liegt zum einen daran, dass die pejorativen Konnotationen von Gräte nicht zu Harfe passen; zum anderen gibt es schon das usuelle Kompositum Harfensaite. Ganz anders ist der Fall bei die Gräten einer Harfe: hier signalisiert die Genitivkonstruktion per se, dass sie vermutlich nicht lexikalisiert ist. Genitivmetaphern können gewissermaßen als „Kreativitätsmarker“ bezeichnet werden: die Konstruktion markiert Okkasionalität und verdeutlicht dadurch, 
dass der kreative Übertragungsprozess gerade und speziell für die jeweilige Textpassage stattgefunden hat. Genitivkonstruktionen sind also gerade deshalb so lyrikaffin, weil sie einerseits für metaphorische Übertragungen in besonderem Maße geeignet sind und andererseits anzeigen, dass eine neue, okkasionelle Bildung vorliegt - beides Merkmale eines kreativen Stils, der für die Lyrik kennzeichnend ist.

\section{Metaphernkomplexe}

Nun unterscheiden sich Metaphern in Gedichten von solchen in nicht-lyrischen Texten unter anderem dadurch, dass man es oft nicht mit Einzelmetaphern zu tun hat, sondern mit Metaphernkomplexen, die mehrere Interpretationsschichten und Mehrfachinterpretationen einzelner Ausdrücke erfordern. Grundsätzlich gilt aber auch hier, was wir über das Auslösen und Lösen von Interpretationskonflikten gesagt haben. Wir wollen das an den ersten beiden Zeilen des Gedichts zeigen, die mit den Gräten einer Harfe enden. Der Ko- und Kontext gibt keine Hinweise darauf, dass es in diesem Gedicht um Harfen geht, wohingegen der Seeteufel im näheren Kotext zunächst suggeriert, dass tatsächlich über Gräten gesprochen wird. Das legt es nahe - im Gegensatz zu der kontextfreien Interpretation des Ausdrucks in Abschnitt 4 (Gräten einer Harfe als 'Saiten einer Harfe') die Harfe in Bezug auf die Fischanatomie als das Skelett des Fisches zu verstehen (Uminterpretierter Ausdruck: Harfe; Konfliktauslöser: Gräten; Konfliktlöser: Gräten als Verweis auf den Bereich FISCHANATOMIE) (s. Abb. 1). 


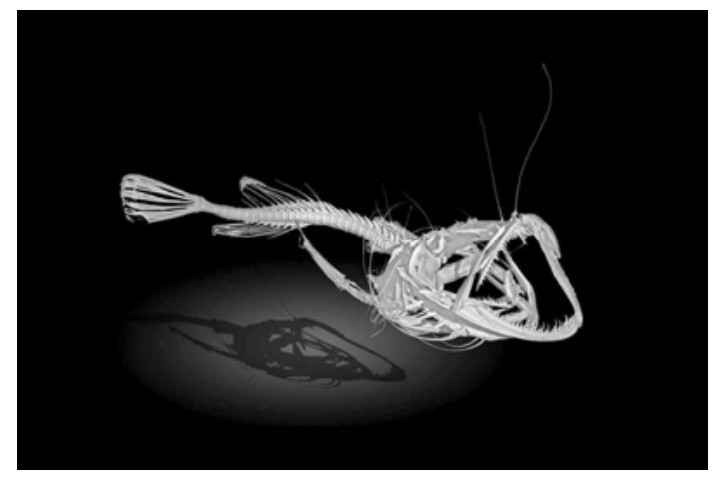

Abb. 1: Skelett eines Seeteufels ${ }^{8}$

Seeteufel selbst ist ein Wort, das Fische der Art Lophius piscatorius bezeichnet (s. Abb. 2). Es ist eine in Form eines Kompositums lexikalisierte Metapher, in der das hässliche, bösartige und gefährliche Aussehen des Fisches an entsprechende Eigenschaften von Teufeln geknüpft wird (Uminterpretierter Ausdruck: Teufel; Konfliktauslöser: See; Konfliktlöser: See als Verweis auf den Bereich MEER/MEERESLEBEWESEN).

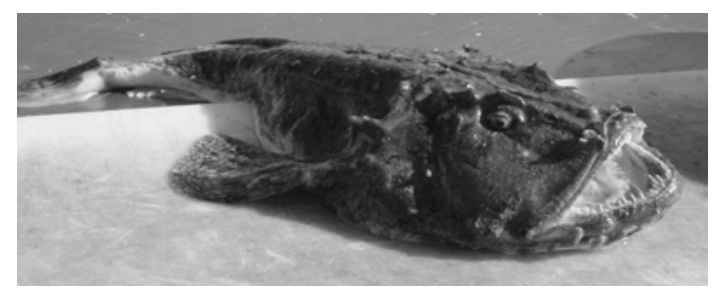

Abb. 2: Seeteufel (Lophius piscatorius) ${ }^{9}$

8 Quelle: Didier Descouens. Online am 19.7.2018:

https://de.wikipedia.org/wiki/Datei:Lophius_piscatorius_MHNT.jpg.

[Creative-Commons-Lizenz: https://creativecommons.org/licenses/by-sa/3.0/deed.de].

9 Quelle: User Meocrisis on de.wikipedia. Online am 19.7.2018:

https://de.wikipedia.org/wiki/Datei:Seeteufel.jpg.

[Creative-Commons-Lizenz: https://creativecommons.org/licenses/by-sa/3.0/deed.de]. 
Nun gibt das Gedicht wenig Anlass anzunehmen, es würde Fische und ihre Anatomie zum Gegenstand haben. Zwar bietet der unmittelbare Kotext weder einen Konfliktauslöser noch einen Hinweis zur Konfliktlösung; jedoch legt der weitere Kontext, etwa die Nennung von Richard III. im Gedichttitel und unser Wissen um den Einleitungsmonolog des Shakespeare-Dramas, es nahe, Seeteufel als Metapher für Richard zu verstehen, um sein hässliches Äußeres zu charakterisieren. Der schon lexikalisierte metaphorisierte Ausdruck für eine Fischart wird gewissermaßen delexikalisiert und auf einer zweiten Stufe metaphorisiert. Während auf der ersten Stufe Konfliktauslöser und -löser innerhalb des Kompositums lagen, müssen sie auf der zweiten Stufe außerhalb des Kompositums gesucht werden (Uminterpretierter Ausdruck: Seeteufel; Konfliktauslöser: Richard III.; Konfliktlöser: intertextuelles Wissen über Shakespeares Drama). Hinzukommt, dass im Zuge einer Deidiomatisierung von Seeteufel auch die Kompositumskonstituente Teufel für eine metaphorische Interpretation frei wird, die ebenfalls eine Interpretation mithilfe intertextuellen Wissens über den Charakter Richards III. erfährt.

In dem Moment, in dem Seeteufel als Metapher für den hässlichen und bösen König verstanden wird, machen die Ausdrücke in seinem Umfeld (zackige Flosse nach oben; transparent die Schuppen; Gräten einer Harfe), für die er nun als Konfliktauslöser fungiert, eine Reihe neuer Metaphorisierungsangebote. Die nach oben gezogene Flosse erscheint nun als Krone (Uminterpretierter Ausdruck: Flosse; Konfliktauslöser: Richard III. als Seeteufel; Konfliktlöser: zackig als Verweis auf GEGENSTÄNDE MIT ZACKEN). Die Gräten einer Harfe, die wir aus strukturellen Gründen zunächst als Saiten einer Harfe, dann - unter Berücksichtigung des „Fisch“-Kontexts - als Gräten am Fischskelett interpretiert hatten, erscheinen nun auch als die Rippen am Skelett des toten Königs (Uminterpretierter Ausdruck: Gräten; Konfliktauslöser: Richard III. als Seeteufel; Konfliktlöser: Richard III. als Verweis auf MENSCHLICHE ANATOMIE) (s. Abb. 3). 


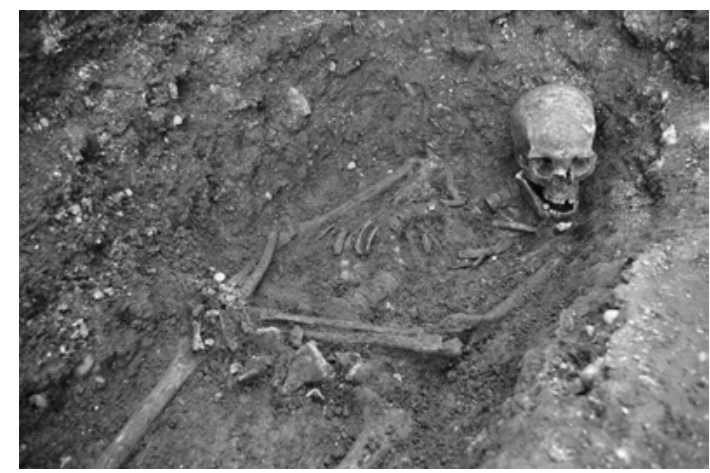

Abb. 3: Gebeine Richards III., die 2012 bei Bauarbeiten in Leicester entdeckt und 2015 in der Kathedrale von Leicester erneut beigesetzt wurden ${ }^{10}$

Und die Frage, ob nun auch die Harfe eine weitere Metaphorisierung verträgt, in der ihre Eigenschaft, Kunstprodukte hervorzubringen, in einen neuen Zielbereich übertragen wird, sei dem Leser hier als Hausaufgabe mitgegeben (Lösung bitte nicht einschicken).

\section{Literaturangaben}

Bade, Nadine \& Sigrid Beck. 2017. Lyrical texts as a data source for linguistics. Linguistische Berichte 251. 317-356.

Joubert, Marlise (Hg.). 2014. in a burning sea. Contemporary Afrikaans Poetry in Translation. Pretoria: Protea Book House.

Knowles, Murray \& Rosamund Moon. 2006. Introducing Metaphor. London \& New York: Routledge.

Lakoff, George \& Mark Johnson. 1980. Metaphors We Live By. Chicago \& London: The University of Chicago Press.

Levin, Samuel R. 1965. Internal and External Deviation in Poetry. In: Word 21(2). 225-237.

10 Seite „Richard III. (England)“. In: Wikipedia, Die freie Enzyklopädie. Bearbeitungsstand: 21. Juli 2018, 14:15 UTC.

URL: https://de.wikipedia.org/w/index.php?title=Richard_III._(England)\&oldid=179332080 (Abgerufen: 25. Juli 2018, 06:02 UTC). Quelle Foto: File:The King In The Car Park - Page 15 - Figure 12.png. (2017, December 21). Wikimedia Commons, the free media repository. Retrieved 16:18, July 24, 2018 from https://commons.wikimedia.org/w/index.php?title=File:The_King_In_The_Car_Park__Page_15_-_Figure_12.png\&oldid=273416346. 
Lindauer, Thomas. 1995. Genitivattribute. Eine morphosyntaktische Untersuchung zum deutschen DP/NP-System. Tübingen: Niemeyer.

Rapp, Irene. In Vorb. Metonymie, Metapher, Personifikation: Uminterpretationen zwischen Semantik und Pragmatik.

Rolf, Eckard. 2005. Metapherntheorien. Typologie - Darstellung-Bibliographie. Berlin \& New York: De Gruyter.

Schuster, Britt-Marie. 2017. Abweichen als Prinzip. In: Anne Betten, Ulla Fix \& Berbeli Wanning (Hgg.), Handbuch Sprache in der Literatur, 310-329. Berlin \& Boston: De Gruyter.

Tawada, Yoko, Günter Blamberger \& Marta Dopieralski (Hgg.). 2018. Die Kunst der Verwandlung/Beyond Identities: poetica 4. Festival für Weltliteratur. Tübingen: konkursbuch Verlag Claudia Gehrke.

Stefan Engelberg ist Leiter der Abteilung „Lexik“ am Institut für Deutsche Sprache (Mannheim), Professor für germanistische Linguistik an der Universität Mannheim und Honorarprofessor an der Universität Tübingen. Er hat an der Universität Wuppertal promoviert und habilitiert. Seine Forschungsschwerpunkte liegen in den Bereichen Lexikologie, Semantik, Syntax-Semantik-Schnittstelle, Lexikographie, Wortbildung, Sprachkontakt, Sprache und Kolonialismus sowie Sprache und Lyrik.

Irene Rapp ist Professorin für germanistische Linguistik an der Universität Tübingen. Sie befasst sich mit Themen zwischen Wort und Diskurs (Wortbildung, Syntax, Wort- und Satzsemantik, pragmatische Inferenzen). Zur Zeit forscht sie insbesondere $\mathrm{zu}$ Tropen in der Lyrik und $\mathrm{zu}$ pragmatischen Inferenzen in Dramentexten. Privat betreibt sie eine Heimwerkstatt zum Video- und Audioschnitt von „Sprachkunstwerken“. 